\title{
Capturing and Documenting Creative Processes in Contemporary Dance*
}

$+$

\author{
Claudia Ribeiro \\ FCSH - Universidade Nova de Lisboa \\ Av. de Berna $26 \mathrm{C}$ \\ Lisboa 1069-061 \\ claudia.ribeiro@fcsh.unl.pt
}

\author{
Rafael Kuffner dos Anjos \\ FCSH - Universidade Nova de Lisboa \\ Av. de Berna $26 \mathrm{C}$ \\ Lisboa 1069-061 \\ rafael.kuffner@fcsh.unl.pt
}

\author{
Carla Fernandes \\ FCSH - Universidade Nova de Lisboa \\ Av. de Berna $26 \mathrm{C}$ \\ Lisboa 1069-061 \\ carla.fernandes@fcsh.unl.pt
}

\begin{abstract}
The performing arts, and dance in particular, have been considered as intangible cultural heritage by UNESCO since 2003. This acknowledgement reflects the importance of preserving the knowledge generated within this art form for future generations. Nevertheless, what and how this sensible material should be preserved is still lacking clear methodological approaches. When considering creative processes, this seems an even more daunting task, as it goes beyond simply documenting the final product of a creation. Recent advancements in technology has allowed to consider other approaches of capturing data apart from video or photography, which are mostly static and have a single viewpoint. In this paper we describe how 3D data capture and point cloud visualization techniques have been used to capture and document João Fiadeiro's choreographic and compositional processes. Together with Fiadeiro we have identified a sub-set of core concepts of his method which have then been used to conduct two improvisation sessions involving Fiadeiro's dancers and himself. Those concepts have been used as the basis for the development of new visualization techniques that better illustrate, in an interactive system, the complexity of Fiadeiro's creative process.
\end{abstract}

\section{CCS CONCEPTS}

-Applied computing $\rightarrow$ Performing arts; •Computing methodologies $\rightarrow$ Point-based models;

\section{KEYWORDS}

Creative Processes, Contemporary Dance, Composition in Real Time, Motion Capture, Point Clouds

\section{ACM Reference format:}

Claudia Ribeiro, Rafael Kuffner dos Anjos, and Carla Fernandes. 2017. Capturing and Documenting Creative Processes in Contemporary Dance. In Proceedings of ACM 4th conference on movement computing, London, UK, fuly 2017 (MOCO'17), 7 pages.

DOI: $10.475 / 123 \_4$

*Produces the permission block, and copyright information

${ }^{\dagger}$ The full version of the author's guide is available as acmart.pdf document

Permission to make digital or hard copies of part or all of this work for personal or classroom use is granted without fee provided that copies are not made or distributed for profit or commercial advantage and that copies bear this notice and the full citation on the first page. Copyrights for third-party components of this work must be honored. For all other uses, contact the owner/author(s).

MOCO'17, London, UK

(C) 2017 Copyright held by the owner/author(s). $123-4567-24-567 / 08 / 06 \ldots \$ 15.00$ DOI: $10.1145 / 3077981.3078041$

\section{INTRODUCTION}

Over the last decade there has been an increase of interest, both from practitioners and from scholars, in the processes of documenting, archiving, and preserving dance [9]. Nevertheless, due to the intricate nature of dance, considerations of what is acknowledged as valuable as a dance object has been illusive [12]. While some believe that the preservation of choreographic works is vital in the development of dance's historical context, others suggest that the ephemeral nature of dance does not lend itself to being preserved at all [14].

A choreographer's legacy is both a historical record that resides in an archived form and a cultural memory or experience that the choreographer envisions to leave behind for future audiences, educators, and scholars [15]. Historically, one of the ways in which Western theatrical dances have been preserved is through the act of score-writing. Despite the efforts of dance-notation practitioners to preserve the art of score-writing, in the latter half of the 20th century, traditional notation systems have become overshadowed by videos as a mode of documenting dances. As technological advancements have continued to alter the practice of dance preservation, a body of literature has emerged that illuminates alternative forms of documentation [11].

Current practices for documentation and preservation are mostly focused on the development of an archive that in itself holds a structure that mirrors a choreographer's life work. In particular, it takes into account the best practices in photo and video digitization and the development of appropriate metadata standards to catalog each component of the archive material. Other relevant considerations are related to the development of a system that enhances humancomputer interaction between the user and the archive. These are all significant issues that should not be neglected. Nevertheless, there still is a lack of work that targets specifically the documentation of creative processes, namely by focusing on the development of a conceptual framework where the evolution of a choreographer's imagistic universe, and how it is translated into his/her aesthetic vocabulary, can be understood.

Inspired by advances in 3D data capture and point cloud visualization techniques, in this paper we describe how these techniques were used to capture and document the artistic work of João Fiadeiro, a Portuguese choreographer. Over the course of two decades, Fiadeiro has been developing a method he calls Composition in Real Time, Composio em Tempo Real, in Portuguese (CTR), which allows him to transmit his artistic modus operandi to his collaborators and to teach a series of compositional principles in international 
workshops and master classes [13]. Together with Fiadeiro, we have identified a sub-set of five core concepts of his method (out of an inventory of 72 terms and expressions), which have then been used as starting-points of two improvisation sessions involving Fiadeirofis dancers and himself. Moreover, those same concepts were also the basis underpinning the development of new visualization techniques that better illustrate in an interactive system how these concepts influence the resulting final performance.

\section{DOCUMENTING AND PRESERVING CONTEMPORARY DANCE}

The way humans perceive and experience art, culture, and heritage in general, perhaps even the idea of the past itself, has already significantly changed with the introduction of smart technologies and innovative applications into our daily lives. Digital technologies do not bring only new tools for access to information, but they should provide new ways of retrieving, connecting, visualizing and understanding the data, and hence possible provide new ways of thinking.

In order to better understand and inform the present by richer interpretations of the past, several actions are being taken at a global level to create affordable and efficient digital access, documentary methods analysis, and preservation services for cultural resources.

In the case of the most ephemeral and intangible art forms, such as contemporary performing arts and dance in particular, the interest in their documentation is even greater precisely because this ephemeral performative action is the ideal territory for working within a fertile nexus of mediations. Each performance brings into play an extended series of instances: creative, technical, material, and societal. In addition, they are linked to research and education materials which also produce a vast amount of associations and may be linked to a myriad of data points. The material generated and the theoretical conclusions reached allow the exploration of new work based on existing information systems, infrastructures, and services in order to make that content available for researchers and educators in a wide range of contexts. As embodied and ephemeral practices, the performing arts comprise a fusion of cultures and practices, but their fluidity within and across borders has been characterized by a dispersed and fractured documented history. Perhaps because, in general, they are heterogeneous practices where the body is the most complex instrument to 'write down', the performing arts have produced very little in what regards "hard-copy records of themselves" [19]. However, this is changing enormously, both concerning the range of methods and systems currently being explored to document performing arts heritage, and in relation to the newly invigorated, creative industry initiatives within Europe.

Slightly over the last ten years, performing artists and researchers in Europe, the USA, and Australia have begun to collaborate in order to explore how digital technologies can offer new methods for documenting dance, theater, and performance in general and for increasing access to these more intangible art forms. These collaborations have resulted in novel ways to preserve and/or reconstruct existing pieces and the creative processes behind them. This is an important moment in time for performing artists in particular: by bringing together different cultures, different practices, and different disciplines, they have a significant opportunity to shape the narrative around their works with a view to creating the optimum framework for its sustainable support into the future. Moreover, recently a number of internationally known choreographers and institutions (e.g. Wayne McGregor - Random Dance, Siobhan Davies in the UK; Emio Greco-PC in the Netherlands, Rui Horta Stage Works in Portugal; the Forsythe Foundation, the Pina Bausch Foundation and the Digitaler Atlas Tanz initiative in Germany) have been proving that it is now essential for contemporary dance and performance to worry about its preservation over time. Other art forms are documented and preserved in their own medium such as painting, sculpture, and film. In the case of music and theatre, there exist universally readable documents in the form of musical notation scores and the dramaturgicscript. Contemporary dance and performance, on the other hand, must develop new forms of mediation and produce new types of resources that make choreographic/compositional ideas more accessible to be studied and understood. The varieties of information-rich resources they have created (including on-line scores, dance digital archives, choreographic software agents and real-time training simulations) confirm their increasing investment in the digital preservation of contemporary creations, an interest which was previously absent [10].

There have been until now different large-scale initiatives at the European level, such as Europeana [7], ECLAP [3] (an e-library for the performing arts), and some other specific artist-drive ones, such as the Siobhan Davies company's "RePlay Archive" [4, 19], where it is possible to navigate online not only through a life-long career of choreographic pieces, but through a catalog of early rehearsal footage and, where available, of filmed records of choreographies in the studio, including original rehearsal materials. The "Pina Bausch Foundation", founded after her death, is also preparing the much expected "An Invitation from Pina: The Pina Bausch Archive" [5], which has developed rather ground-breaking ideas on what dance and theater documentation can be about, aside from the foundation's" more institutional archival initiatives. Also, the "K3 Zentrum für Choreographie" [8] has an ongoing cooperation project called "Reflex Europe" which is focused on studying how documenting the creation process can improve learning in the context of contemporary dance.

To conclude this listing, "Motion Bank" [1] was the newest Forsythe Company project (after their highly successful project "Synchronous Objects" [6]), launched in 2013, and having provided a broad context for research into choreographic practice. Their main focus was on the creation of on-line digital scores in collaboration with four guest choreographers of broad international projection. However, they do not aim at an open-ended platform to accommodate inter-relations and collaborative resources between performing artists in general, as accomplished with the "Transmedia Knowledge-Base for performing arts [2], developed between 2011 and 2013, and officially launched online in June 2016.

Technologies developed for the purpose of analyzing and archiving movement in creative areas such as dance and performance are opening up new ways of exploring and reactivating specialist gestural knowledges from the past [16]. In this manner, contextualized simulations and re-enactments of corporeal movement are providing insights into gestural skills that are otherwise being rapidly subsumed and forgotten in the wake of technological developments 
- notably those involving digitization and manual or semi-automatic video annotations.

\section{COMPOSITION IN REAL TIME}

Since 1995, Fiadeiro has continued to develop his Composition in Real Time (CTR) method. At first, the system was established to collaborate with fellow artists to successfully create coherent stage works. As the method was systematized over the years, not only an increasing number of artists were engaging with the CTR, but non-performers as well, such as theoreticians, academics, and researchers from such diverse fields as the social sciences (philosophy, anthropology, ethnology), economics, complex system theory, and neurosciences. The dialogue with these experts in turn helped to enrich the CTR into a full fledged compositional system, which principles can be applied in different artistic and scientific processes.

At the heart of the CTR lies a novel approach to the decisionmaking process. Fiadeiro observed early on in dance improvisation that performers would not dedicate as much time to evaluating their possibilities to act in a certain set of circumstances. The CTR method suggests to suspend the impulse to act immediately upon the situation one finds oneself in. Instead the performer takes the time needed to better understand the properties and possibilities inherent to the situation to make an informed decision. This decision results in an (performative) action, which is called a 'position' in the CTR. Another performer will subsequently perform the next action (or position), also using the time during suspension to make a decision which relates to the action (or position) of the performer before. In other words, a relation between the two positions (actions) is created. The third action taken by the next performer should relate to the relation established by the two actions before, so that a clear direction for the improvisation is proposed. During the cycle of vitality this direction is explored, until the improvisation comes to a natural end that all performers agree upon.

To put it simply, all participants in an emerging compositional process act collaboratively, building something by relating to each others' actions. By working in this interrelated way, it becomes evident for the participants that many possibilities exist at each moment of decision, which can be seen as "possible futures" (and pasts, for that matter). Whenever a decision has been made, the past is retroactively rewritten, that is, interpreted in a new way. Particularly this latter concept has been attracting much attention by researchers from the above-mentioned scientific domains.

\section{CASE STUDY}

The main aim of our case study was to capture and visually represent a sub-set of the most representative concepts of the Real-Time Composition method. In order to do so, we captured two improvisation sessions with João Fiadeiro and seven of his dancers in two separate days. Each session had an approximate duration of three hours, and each improvisation started with the same position.

\subsection{Composition in Real Time Concepts}

Fiadeiro has devised a system representing his own creative process in which the underlying fundamental concepts are not static; they are organic and evolve as Fiadeiro himself transforms his own

\begin{tabular}{l|l} 
CTR Concept & Description \\
\hline Position & $\begin{array}{l}\text { Any performed act/ A performative act. } \\
\text { Example: a performer lifts a chair. }\end{array}$ \\
\hline Relationship & $\begin{array}{l}\text { Performing a position which relates to } \\
\text { another position. Example: another per- } \\
\text { former also lifts up a chair. }\end{array}$ \\
\hline Suspension & $\begin{array}{l}\text { The time a performer takes to make a } \\
\text { decision and suspends any action. }\end{array}$ \\
\hline Cycle of Vitality & $\begin{array}{l}\text { The time or duration of an improvisa- } \\
\text { tion exploring a specific idea. }\end{array}$ \\
\hline Possible Futures and & $\begin{array}{l}\text { At each moment in the improvisation, a } \\
\text { performer can choose from several pos- } \\
\text { sibilities of how to proceed (= futures), } \\
\text { which will shape reinterpretations of } \\
\text { past understandings of positions. }\end{array}$ \\
\hline
\end{tabular}

Table 1: Summary of five core Composition in Real Time concepts.

choreographic perspectives and practices based on new experiences and projects. He has also devised a version of his game to be practiced by people not accustomed to using their bodies in the same way as dancers, where objects are used on a table instead of bodies on a dance floor. In order to acquire sufficient knowledge of his method, we actively and passively participated in several workshops, including table versions and more specialized workshops targeted at expert dancers.

Following these sessions, it was important to understand how these core concepts of the CTR method related to the CTR system, as well as to the final result of an improvisation session. In particular, we wanted to understand if it were possible to visually represent his concepts using improvisation sequences from Fiadeiro's current works. With this aim, we conducted several unstructured interviews with Fiadeiro where we first focused on identifying a set of core concepts. From these interviews resulted the selection of five concepts described in Table 1.

The next stage focused on observing videos of Fiadeiro's past works, including rehearsals, performances, and workshops, and identifying a set of video sequences corresponding to each concept. This allowed us to have both a conceptual understanding as well as a visual representation of the concepts. We have then together with Fiadeiro developed a set of possible visualization effects for each concept. Final results are described in Section 5.

\subsection{Motion Capture and Point Cloud Visualization}

From the participation and observation of Fiadeiro's workshops, it was clear that space was a limitation, meaning we had to considered how many Microsoft Kinects would be necessary, and what was the ideal setup to guarantee data quality. Other aspects to consider when using multiple Kinects are data calibration and synchronization. 
To guarantee the capturing of the complete improvisation session, a wide-baseline setup was used, where each view was captured by a Kinect sensor. Each view was positioned on opposite sides of the room in order to minimize loss of data due to occlusion (see Fig. 1). Adding more viewpoints decreases the probability of occlusions but also linearly increases the amount of data to be handled during rendering.

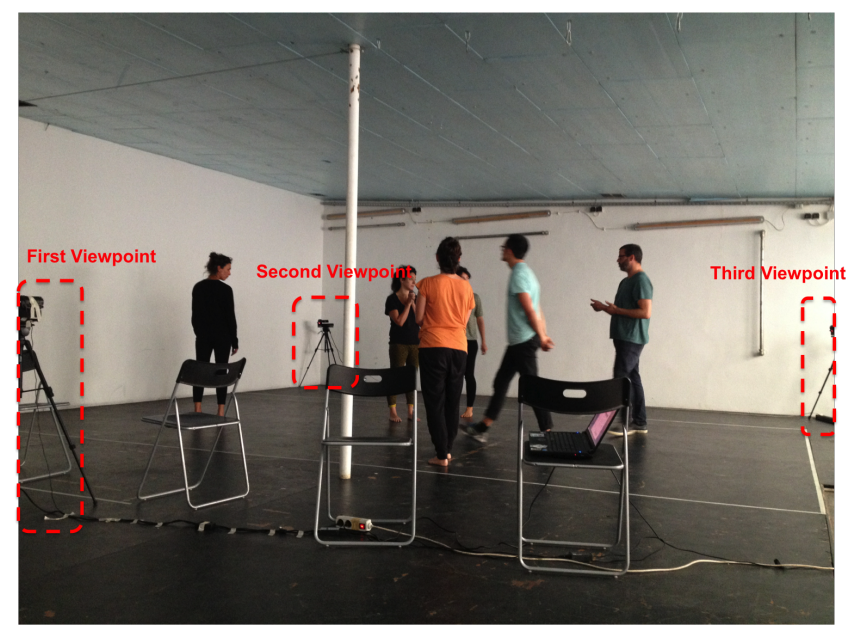

Figure 1: Representation of the setup used with three Kinects and three video cameras during the case-study done with João Fiadeiro and his contemporary dancers.

Regarding data synchronization, a network-based synchronization program was developed allowing to trigger the capture remotely and simultaneously on each computer. The calibration of extrinsic and intrinsic parameters was performed using OpenCV and manual inputs from the developers since the process was performed in a controlled scenario.

The post-processing stage follows the data flow presented in Figure 2. First the color images and depth maps are converted into point cloud data. This type of data are simple Cartesian coordinates and their respective color information, therefore there is no contextual information about what is actually represented in the point cloud. At this stage, it is not yet possible to manipulate individual elements of the point clouds, such as people or objects.

The Point Cloud Library [18] Euclidean Cluster Extraction algorithm was used to separate the point cloud into clusters. This algorithm basically separates unorganized point clouds into separate clusters (sets of points) based on their distances. After the initial tests it became obvious that this information was not enough to clearly separate point clouds into clusters. Specifically, in certain situations the dancers were all concentrated in the same position (e.g. while holding the same object) and only moved slightly, tricking the algorithm to assume they became a different cluster.

To improve cluster detection we adapted the original algorithm by adding another condition to be considered besides distance. First we considered only the first frame, and for each cluster we calculated the AABB box and respective center. We then processed the remaining frames using the distance between points and also

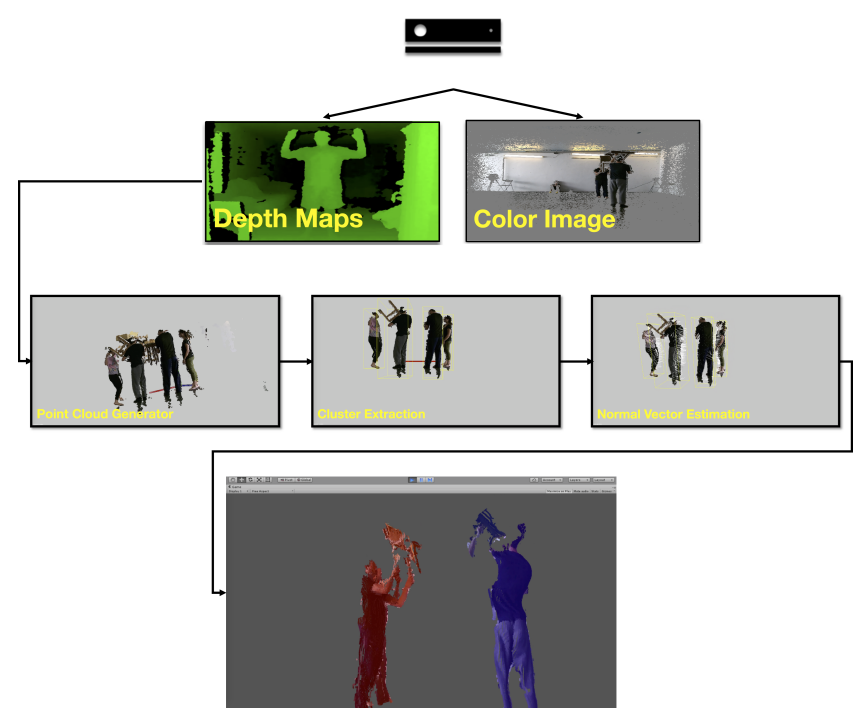

Figure 2: Flowchart of data capture, post-processing, and visualization. Data (depth maps and color images) was captured using a Microsoft Kinect. This was then inputed into an in-house software, which generated the point cloud data. A cluster extraction algorithm was then applied, finishing with the estimation of the normal vector for each point of the point cloud. Finally, the point cloud visualizer was implemented in Unity3D.

verified that the distance to the center of the AABB box of the frame before is below a certain threshold determined during the testing phase.

There are also challenges related to point cloud visualization such as loss of definition on close-ups, foreground/background confusion, and depth perception due to the absence of occlusions between the rendered points. These issues are specially noticeable on low resolution scans or background objects.

Finally, we developed a point cloud visualizer using Unity3D ${ }^{1}$. Within this visualizer we implemented scripts which automatically apply the intended effects on to the point cloud clusters according to annotation data for the concepts of position, relation, and cycle of vitality. The remaining two concepts were implemented as different scenes in Unity3D, since they had to be manually crafted to adapt the contents of the improvisation sessions.

\section{RESULTS}

In order to illustrate each one of the concepts, short sections of data from the improvisation sessions which clearly demonstrated the selected base concepts from CTR were selected.

Since some concepts could only be well perceived by the external users through long periods of time, we combined different subsections in order to offer a shorter but sequenced visualization. To visualize the data we used a point cloud visualizer previously implemented in Unity3D. Each data set used to illustrate a particular

\footnotetext{
${ }^{1}$ https://unity3d.com
} 

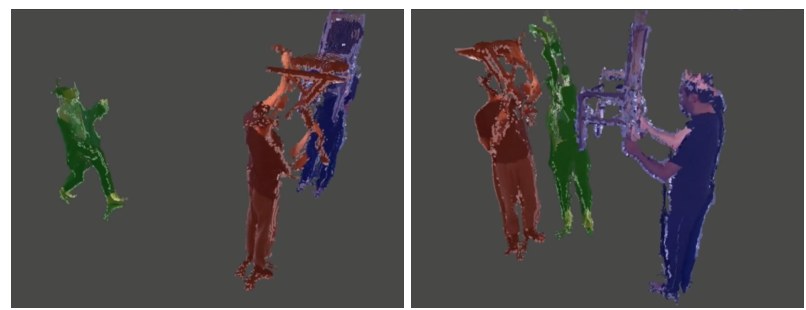

(a) Two shots of the position example. Each participant represents a consecutive position.

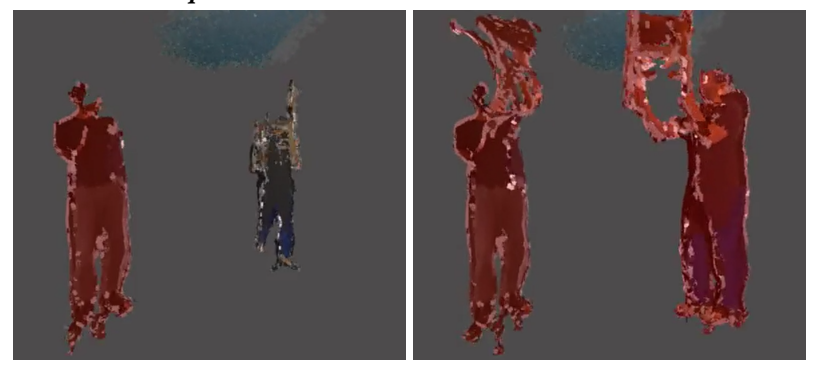

(b) Relation: The second participant slowly assimilates the color of the first as they establish a relation

Figure 3: Color-based representation of CTR concepts.

concept was post-processed, as explained in the previous section, and then read offline by the point cloud visualizer. The software also includes a basic graphical user interface which allows the user to interact with the point cloud, specifically to navigate freely within the 3D scene, fast foward, or view each frame step by step.

The most relevant CTR core concepts, position, was represented through assigning different colors to elements introduced in a certain position, so that users can keep track of the development of the improvisation session. In our chosen segment, each participant entering the stage has created a new position. We have identified each different body through a color-based clustering algorithm, combined with video annotations [17], which would connect each player to a different position and an assigned color.

An example can be seen in Figure 3a, where the third position is being introduced. The participant in red entered the space holding a table in the air (first position) followed by the one in blue, who was holding a table in the air (second position), and by the one in green (third position) with a cup that is held above the table.

Relations formed by different elements introduced in the composition were also identified by the same color. The elements introduced in a new position may create a relation with some or all of the elements from previous positions in the scene. The establishment of newer relations was represented as a gradual assimilation of colors, as seen in Figure 3b. This example only contains a single relation between two elements: the participant holding a chair and the one holding the table, which creates a clearly identifiable relation coming from the real world. In a more complex scenario, several separate relations may co-exist at any given time. Changes in relations and positions can go by unnoticed by novices to this method, specially in longer scenarios, with more performers, or in a faster paced sequence of actions. Albeit a simple solution, color coding can clearly contribute to identifying these concepts without adding visual pollution to the actual recorded content.

The Cycle of vitality, i.e. the time frame during which the composition is progressing, is exemplified through the loss and gain of color. Following the CTR rationale, the end should always be postponed by allowing the creation of new relations through new positions. We selected a long sequence of a session performed by two participants, where we were able to see the prolongation of the cycle of vitality through several positions, as well as its approach to an end, when no new positions were being introduced (see Figure $4 \mathrm{~b})$. In this particular example, one participant decides to leave the scene as he hurts himself during the performance. The cycle was coming to an end, but another performer introduces a new position by inviting him back to the stage, using the accident as an element of their improvisation, therefore starting a new cycle of vitality from that relation.

The three-dimensional aspect of the captured data allows a better representation of the concept of Suspension, which is used in the decision making process. By spatially separating present and future possible positions and manipulating the reproduction speed of a segment, we exemplify the suspension of time in which one participant is examining the current situation with her personal time being disconnected from the real time. Figure 4a shows our visualization of this concept. In this particular sequence, each position would add objects to the objects pile carried by the participant who had the table in the first position. At a certain moment, a critical point is reached, where it should be considered to finish the current sequence of positions, due to the fact that the first participant was not able to carry more objects. Here a suspension is exemplified, with the reproduction speed of the "present" (participant holding objects) slowed down, to represent the disconnect between real time and the time as perceived by the outermost participant, representing the desired decision making process in CTR. The most likely future possibility can be contemplated in the background, and when that future is "accepted" (each participant implicitly decides this is the course of action to be taken), the camera navigates into the new present scene.

One remarkable feature of Fiadeiro's work is the concept of rewriting the past by changing the future, i.e. by creating new relations. Our last example shows possible futures and pasts. We recorded different improvisation sessions always repeating the first position and giving freedom to the performers from then onwards. We spatially organize the possible futures in a three-dimensional space. The performer is placed inside a cube, in which he has a possible future in each one of the sides of the cube. We navigate the camera through each one of the possible futures for new relations. In reality, there are countless possible futures for each position. We display all the different outcomes from starting at the same first position to exemplify this concept.

\section{DISCUSSION}

Capturing and documenting contemporary dance is in no way a trivial task. This is due to the ephemeral nature of dance, which constitutes a challenge when trying to understand which dance objects are valuable resources for the research community, choreographers and dancers, and the general public. Even though it is currently 

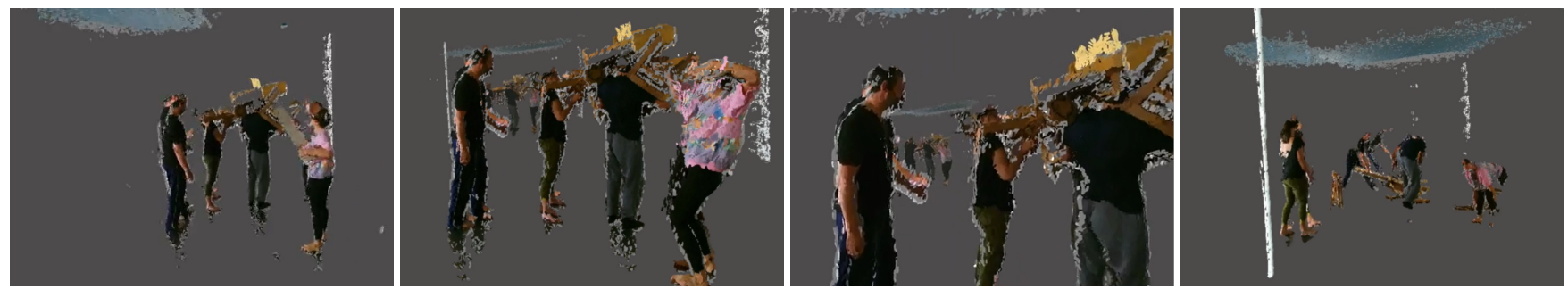

(a) Suspension: Sequence of instances in the visualization where the concept of possible future appears in the background, and the camera navigates in that direction when the suspension ends.
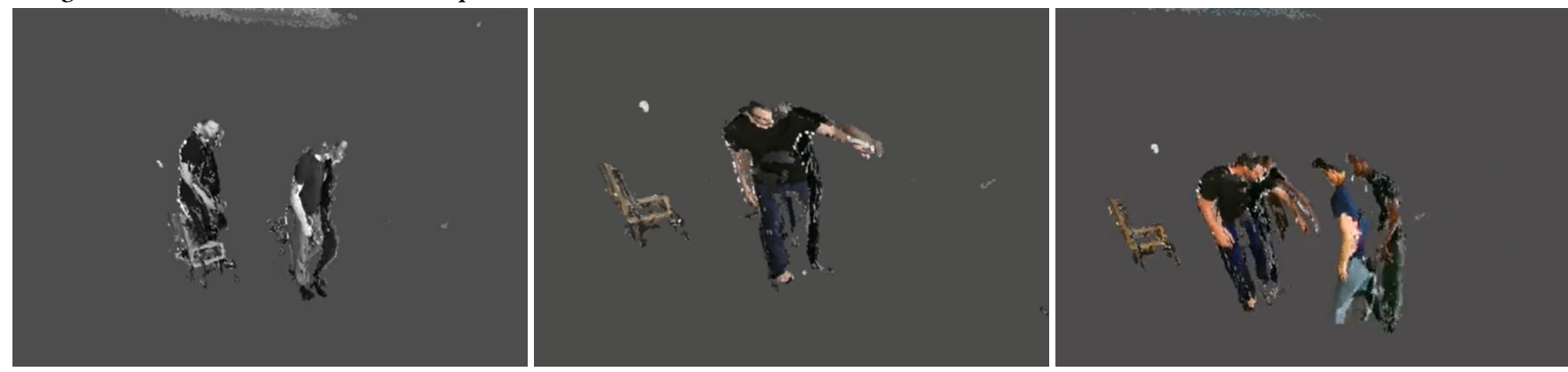

(b) Cycle of vitality: The cycle ends as a performer leaves the stage. Saturation gradually comes back when another performer starts a new position.
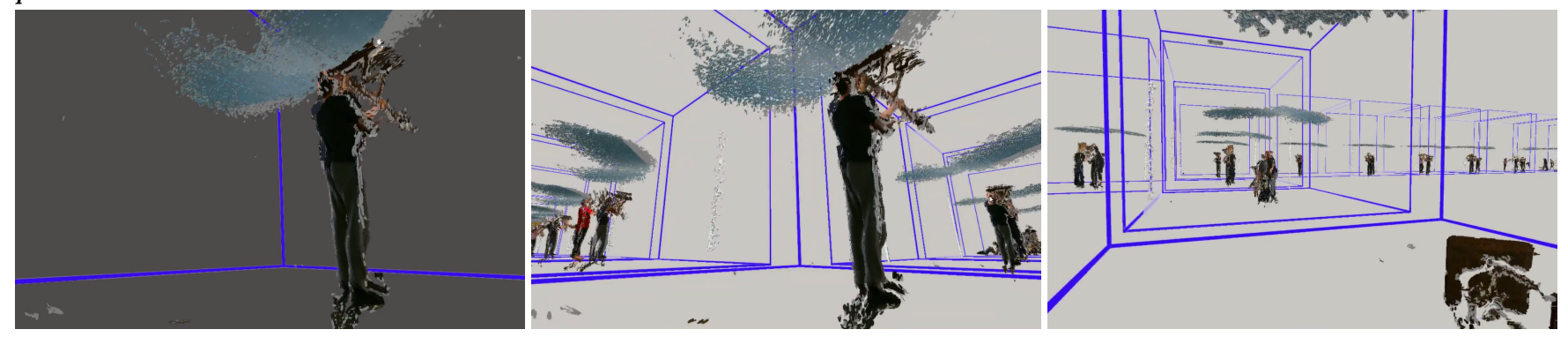

(c) Possible futures and pasts: Possibilities are unveiled through the sides of the cube, where possibilities are organized in a three-dimensional space.

Figure 4: Sequences of the generated visualizations for CTR concepts.

common practice for choreographers or foundations/institutes to maintain some sort of archive of their works (typically comprised of photos, interviews, and videos), the same is not true when considering the documentation of creative processes. Moreover, photos and videos are static content which impose the vision of the person who photographed/recorded and edited the material. Therefore, showing but one perspective of the complex system that composes a choreographic work. These kind of static material also limits the ability to interact, experiment and explore the creative dimensions underlying a choreographic process.

Capturing dance in three dimensions supporting a viewpointfree visualization opens up a vast range of possibilities. Primarily, dance is about movement, specifically about expression through movement. Having an interactive digital representation of movement allows us to closely observe subtle particularities that characterize a particular aesthetic language and, in this manner, preserve choreographic works within the creative context where they had been created. 3D data is a composition of graphical primitives that can be manipulated and changed to serve diverse purposes. By manipulating this kind of data it is possible to provide different viewpoints which can open up new future perspectives related to a choreographer's piece. This was the ultimate goal of our work: to provide an alternative and interactive visualization so that future generations can create their own stories when experiencing Fiadeiros' conceptual work.

Although the proposed visualizations are specific to Fiadeiro's creative process, the methodology used to create them can be applied to other contexts. Annotating three-dimensional point cloud data is a flexible-enough basis to build different effects upon. Among the presented results, the color manipulation effects (Position, Relation, Cycle of vitality) may have a direct application in scenarios with different sets of concepts. Spatial organization of selected parts of a performance can also be used to demonstrate its development or evolution over longer periods of time, comparisons between similar segments, or other types of relevant concepts to the work at hand. 
We should add that in this study we have only taken into account the participation of the choreographer in the design and evaluation of the system, and not the dancers'. In other words, we did not focus on the possible feedback that could be given by the dancers to improve the system, as our main focus was on the process of composition in itself, namely on the particular method of "Composition in Real Time" and not on the usability of the visualizer we have developed.

This technique has its own challenges and limitations which have to be mentioned. In particular, it is necessary to understand how a performance can be enhanced through the use of technology without compromising the dance object. Also, how this type of data can be used in a virtual environment and providing a way for the user to intuitively understand the inspiration and motivations behind that dance object.Although several affordable motion capture systems exist, their use is not at all straightforward.It is still necessary to develop specialized software to deal with this kind of data, which requires software engineers. Moreover, maintaining digitized archives requires specific maintenance and specific hardware that can often be costly.

\section{CONCLUSION}

We have presented an alternative approach to archiving and visualizing the choreographic work of a performing artist, by using the work of João Fiadeiro as a case study. Not only is our data stored as a series of point clouds, which already enables a better spatial perception of the work, but we have also developed meaningful visualizations to illustrate core concepts of Fiadeiro's Composition in Real Time method.

Five different possible visualizations have been presented for this use case by manipulating color, time, and spatial position, and by displaying some of the capabilities of this archiving representation. Moreover, when compared to a simple video, the created visualizations inform viewers of a series of annotated concepts that would not be clear by simply attending the staged performance, thus allowing for the preservation of knowledge behind the full creative process, and not only of its final result.

Improvements on the data representation of the point cloud sequences is the key point to move forward in order to allow a user to watch longer segments of a performance. We will also explore different visualization and rendering techniques that can represent different concepts from other choreographers. The quality of the data will naturally increase with the evolution of laser scan hardware, hopefully allowing the capture of a higher number of performers and with better precision.

\section{ACKNOWLEDGMENTS}

This work was supported by the European Research Council under the project "BlackBox: A collaborative platform to document performance composition: from conceptual structures in the backstage to customizable visualizations in the front-end" (Grant agreement n. 336200). We extend our acknowledgments to João Fiadeiro and the dancers who have participated in this case-study, as well as to the team at Re.Al for their technical support and collaboration.

\section{REFERENCES}

[1] 2010 (accessed Jan 12, 2017). Motion Bank. http://motionbank.org/en.

[2] 2010 (accessed Jan 20,2017). Transmedia Knowledge-Base for performing arts. http://tkb.fcsh.unl.pt/content/knowledge-base-performing-arts.

[3] 2011 (accessed Jan 10, 2017). The ECLAP e-library of performing arts. http: //labs.europeana.eu/apps/eclap.

[4] 2013 (accessed Jan 10, 2017). The Choreographic Archive of Siobhan Davies Dance. http://www.siobhandaviesreplay.com.

[5] 2013 (accessed Jan 10, 2017). The Pina Bausch Archive. http://www.pinabausch. org/en/archive.

[6] 2013 (accessed Jan 12, 2017). Synchronous Objects. http://synchronousobjects. osu.edu.

[7] 2014 (accessed Jan 3, 2017). Europeana Project. http://www.europeana.eu/portal/ en.

[8] 2017 (Accessed: 2017-04-22). K3 fi Centre for Choreography-REFLEX Europe. http://k3-hamburg.de/en/koopprojekte.

[9] Andreas Aristidou, Efstathios Stavrakis, Panayiotis Charalambous, Yiorgos Chrysanthou, and Stephania Loizidou Himona. 2015. Folk Dance Evaluation Using Laban Movement Analysis. Journal on Computing and Cultural Heritage ( FOCCH) 8, 4 (2015), 20.

[10] Scott Delahunta and Norah Zuniga Shaw. 2008. Choreographic resources agents, archives, scores and installations. Performance Research 13, 1 (2008), 131-133.

[11] N Grammalidis, K Dimitropoulos, F Tsalakanidou, A Kitsikidis, P Roussel, B Denby, P Chawah, L Buchman, S Dupont, S Laraba, and others. 2016. The i-Treasures Intangible Cultural Heritage dataset. In Proceedings of the 3rd International Symposium on Movement and Computing. ACM, 23.

[12] Valeria Lo Iacono and David HK Brown. 2016. Beyond Binarism: Exploring a Model of Living Cultural Heritage for Dance. Dance Research 34, 1 (2016), 84-105.

[13] Stephan Jürgens, Francisco Henriques, and Carla Fernandes. 2016. ReConstructing the Choreographic Studio of João Fiadeiro Through Animated Infographic Films. PARtake: The fournal of Performance as Research 1, 1 (2016), 3.

[14] Barbara Kirshenblatt-Gimblett. 2014. Intangible Heritage as Metacultural Production. Museum International 66, 1-4 (2014), 163-174.

[15] Cheryl LaFrance. 2011. Choreographersfi Archives: Three Case Studies in Legacy Preservation. Dance Chronicle 34, 1 (2011), 48-76.

[16] S.J. Norman. 2016. Dancing on Occam's Razor: Expressive Movement and Place. In Multimodal Communication in Language, Performance and Digital Media, C. Fernandes (Ed.). Cambridge Scholars Publishing, Newcastle upon Tyne, 82-94.

[17] Claudia Ribeiro, Rafael Kuffner, Carla Fernandes, and João Pereira. 2016. 3D Annotation in Contemporary Dance: Enhancing the Creation-Tool Video Annotator. In Proceedings of the 3rd International Symposium on Movement and Computing (MOCO '16). ACM, New York, NY, USA, Article 41, 4 pages. DOI : http://dx.doi.org/10.1145/2948910.2948961

[18] Radu Bogdan Rusu and Steve Cousins. 2011. 3D is here: Point Cloud Library (PCL). In IEEE International Conference on Robotics and Automation (ICRA). Shanghai, China.

[19] Sarah Whatley. 2013. Siobhan Davies RePlay:(Re) visiting the digital archive. International Fournal of Performance Arts and Digital Media 9, 1 (2013), 83-98. 